\title{
Le corps médical est ouvert à de nouveaux modèles de financement
}

\author{
Beatrix Meyer ${ }^{a}$, Cloe Jans ${ }^{b}$, Lukas Golderc \\ ${ }^{a}$ cheffe de la division Médecine et tarifs hospitaliers, FMH; ${ }^{b}$ cheffe de projet gfs.bern; ${ }^{c}$ co-directeur gfs.bern
}

Les médecins favorisent un financement uniforme des secteurs ambulatoire et hospitalier qui permettrait de réduire les incitatifs négatifs. Par ailleurs, la hausse permanente du temps consacré à la documentation impacte de plus en plus les médecins: par rapport à 2011, les médecins dans les hôpitaux de soins somatiques aigus passent en moyenne 26 minutes de plus par jour à documenter les dossiers.

Les hôpitaux de soins somatiques aigus facturent depuis six ans avec le système de forfaits par cas SwissDRG. Les prestations de psychiatrie hospitalière de l'adulte seront indemnisées à partir de début 2018 avec TARPSY. L'introduction du système tarifaire ST Reha est prévue en 2020 pour la réadaptation hospitalière. De plus, plusieurs changements importants du système de financement de la santé publique en $\mathrm{Su}$ isse sont en discussion. Afin de détecter suffisamment tôt d'éventuelles évolutions négatives, l'institut de recherche gfs.bern mène depuis 2011 une enquête annuelle représentative sur mandat de la FMH. Quelque 1471 médecins ont participé à la dernière édition de l'enquête cet été 2017. ${ }^{1}$

\section{Favorable à un financement uniforme hospitalier - ambulatoire}

Franchise et quote-part exceptées, les prestations ambulatoires sont à la seule charge des assureurs-maladie. En revanche, dans le secteur hospitalier, les cantons participent aux coûts des prestations fournies dans les hôpitaux de leur liste hospitalière à hauteur d'au moins 55\%. Par conséquent, un patient hospitalisé est souvent meilleur marché pour les assureurs qu'un patient traité ambulatoirement. Cela crée donc des incitatifs négatifs qui rendent le système de santé globalement plus coûteux. Les médecins hospitaliers de soins somatiques aigus ont relevé dans leur domaine

\section{Nouveaux modèles de financement}

Pour de plus amples informations relatives aux modèles de financement: www.fmh.ch $\rightarrow$ Tarifs hospitaliers $\rightarrow$ Publications $\rightarrow$ Séance de la FMH: nouvelles pistes de financement dans le système de santé. d'activité que la décision de traiter une personne en ambulatoire dans leur institution sans l'hospitaliser n'était pas prise selon des motifs médicaux en moyenne 4,4 fois par mois. De plus, une majorité relative des médecins interrogés n'est pas satisfaite du finance-

Les médecins souhaitent le cofinancement du secteur ambulatoire par les cantons.

ment actuel. L'attente de nouvelles solutions est donc nettement perceptible, mais la tendance la plus forte, et ce de loin, va vers un financement uniforme des prestations ambulatoires et hospitalières. Les cantons participeraient ainsi de la même manière aux secteurs ambulatoire et hospitalier, par exemple avec une part de financement à hauteur de $25 \%$. 32\% des médecins ambulatoires (cabinets) et 37-44\% des médecins hospitaliers des domaines de la réadaptation, des soins somatiques aigus et de la psychiatrie approuvent cette solution. Un financement moniste par les assureurs seuls est en revanche tout aussi peu accepté que le modèle d'aujourd'hui. Une personne interrogée sur six n'est convaincue par aucune de ces solutions ou défend d'autres idées. Près d'un quart est indécis ou ne s'est pas prononcé (cf. fig. 1).

\section{Toujours des réserves vis-à-vis de SwissDRG et TARPSY}

Si une majorité relative du corps médical souhaite d'autres modèles de financement, c'est-à-dire un changement de celui qui paie la facture de l'hôpital, les médecins continuent en revanche d'émettre des réserves quant aux systèmes tarifaires. Près de la moitié des médecins concernés se déclarent clairement ou plutôt 
"Quel système de financement souhaitez-vous le plus pour l'avenir?" en $\%$ de médecins ambulatoires/soins somatiques aigus/psychiatrie/réadaption

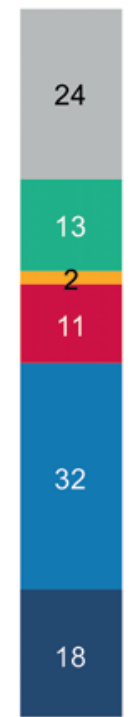

Médecins ambulatoires

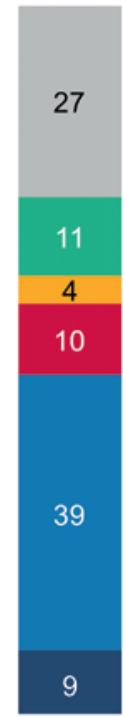

Médecins en soins somatiques aigus

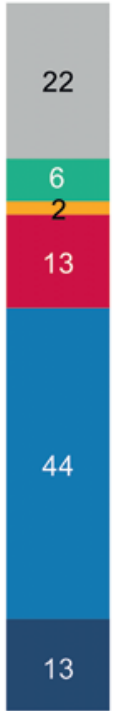

Psychiatrie

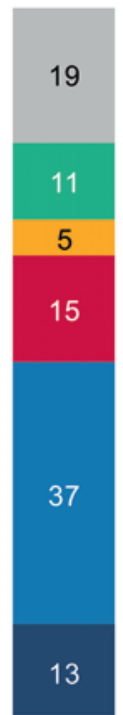

Réadaption
- Ne sait pas/pas d'indication

- Aucune de ces idées n'est convaincante

\# Autres

- Le financement soit pris en charge uniquement par les assureurs dans les secteurs ambulatoire et stationnaire

- Les cantons participent au financement du secteur stationnaire et aussi à celui du secteur ambulatoire

- Le même qu'aujourd'hui

๔ gfs.bern, Etude concomitante effectuée sur mandat de la FMH, juin - août 2017 ( $\mathrm{N}$ médecins ambulatoires = 295 / $\mathrm{N}$ médecins en soins somatiques aigus $=974 / \mathrm{N}$ psychiatrie $=127 / \mathrm{N}$ réadaption $=75)$, sig.

Figure 1: Système de financement souhaité.

contre les systèmes tarifaires SwissDRG et TARPSY. Moins de réserves sont émises en réadaptation: près d'un quart des médecins de réadaptation hospitalière se déclarent clairement ou plutôt contre l'introduction du système ST Reha. Mais beaucoup ne se sont pas encore forgé d'avis à ce sujet, cela peut éventuellement venir du fait que la forme exacte de la version d'introduction de ST Reha n'est pas encore connue.

\section{Davantage de documentation - moins de temps pour les patients}

Le temps consacré à la documentation des dossiers impacte de plus en plus les médecins. Les médecins hospitaliers de soins somatiques aigus y passent en moyenne 19\% de leur temps de travail (cf. fig. 2), soit 112 minutes par jour; ce sont 26 minutes de plus qu'en 2011. En psychiatrie, ce taux est de $15 \%$ et de $18 \%$ en réadaptation. Par conséquent, il reste de moins en moins de

\section{Modèles de documentation}

Pour simplifier la documentation, la FMH a élaboré des modèles pour documenter les codes complexes. II en existe désormais aussi pour la psychiatrie: www.fmh.ch $\rightarrow$ Tarifs hospitaliers $\rightarrow$ SwissDRG $\rightarrow$ Modèles de documentation. temps pour les patients: en soins somatiques aigus et en psychiatrie, les activités au chevet du patient ne constituent plus qu'un tiers, et en réadaptation plus qu'un cinquième du temps de travail. Les travaux de documentation concernent en première ligne les médecins-assistants: en soins somatiques aigus, ils passent $28 \%$ de leur temps de travail à la documentation et seulement 30\% à des activités auprès des patients.

\section{Baisse de la satisfaction au travail malgré une forte identification professionnelle}

La grande majorité des médecins s'identifient fortement à leur profession et apprécient le travail en équipe, notamment aussi avec le personnel infirmier. Près de $80 \%$ des médecins hospitaliers en soins somatiques aigus et en psychiatrie sont satisfaits de leur activité professionnelle. En réadaptation, ils sont $75 \%$. La satisfaction est la plus élevée (87\%) chez les médecins en cabinet. Soulignons cependant que ces taux sont en baisse depuis 2011 dans tous les domaines précités, et plus particulièrement en psychiatrie et en réadaptation avec une baisse de près de 10 points. La satisfaction est souvent entravée par une forte pression liée au temps et à la performance mais, parfois 
"Si vous pensez à votre dernière journée de travail normale, combien de temps avez-vous consacré aux tâches suivantes? Veuillez fournir des réponses en minutes."

Aux travaux liés à la documentation médicale/dossiers de patients/rédaction de rapports

En \%, valeurs moyennes, différentes tâches pour une journée de travail complète, médecins en soins somatiques aigus
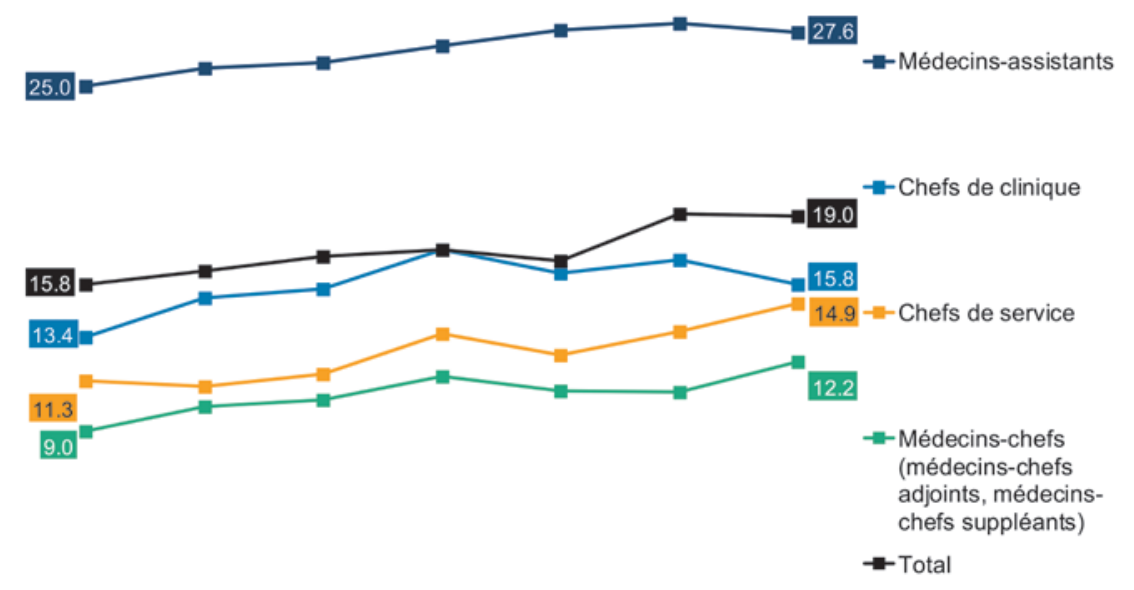

$\begin{array}{llllll}2011 & 2012 & 2013 & 2014 & 2015 & 2016\end{array}$

(C) gfs.bern, Etude concomitante effectuée sur mandat de la FMH, juin - août 2017 (N médecins en soins somatiques aigus env. 940)

Figure 2: Temps consacré à la documentation médicale.

aussi, par une mauvaise organisation du travail à l'hôpital. Cette dernière est pointée du doigt par 36\% des médecins hospitaliers. Les heures supplémentaires s'élèvent en moyenne à 7,4 heures par semaine pour les postes à plein temps. En soins somatiques aigus et en réadaptation, la part de médecins hospitaliers qui envisagent un poste en dehors de notre système de santé en Suisse est nettement plus élevée qu'en 2013. Aujourd'hui, elle est déjà de $16 \%$ en soins somatiques aigus, de $17 \%$ en réadaptation et de $9 \%$ en psychiatrie hospitalière. Ce taux reste constant à $6 \%$ pour les cabinets médicaux.

\section{Bonus de plus en plus répandus en psy- chiatrie et en réadaptation}

Près d'un tiers des médecins hospitaliers ont une part variable dans leur rémunération. Il s'agit en premier lieu de bonus liés à la performance mais aussi d'honoraires d'une activité médicale privée. $9 \%$ des médecins hospitaliers en soins somatiques aigus perçoivent un bonus lié à la performance. Ce taux a légèrement diminué par rapport aux deux années précédentes. En revanche, la part de bonus lié à la performance est en hausse en psychiatrie et en réadaptation, avec $14 \%$ pour la première et $15 \%$ pour la seconde (cf. fig. 3 ). Pour autant qu'un médecin hospitalier perçoive un tel bonus, ce dernier correspond en moyenne à 17\% du revenu total en soins somatiques aigus alors qu'il était encore de $20 \%$ en 2013. Les contrats stipulant des bonus liés à la performance pour un médecin hospitalier peu-

Les bonus des médecins hospitaliers en soins somatiques aigus ont diminué.

vent se révéler problématiques lorsque les objectifs à atteindre fixent des nombres de traitements. ${ }^{2}$ Pendant un mois, les médecins hospitaliers interrogés (soins somatiques aigus) ont relevé dans leur environnement professionnel une moyenne de 1,5 opération et de 2,3 traitements non opératoires médicalement non indiqués. Mais ils ont aussi noté que deux traitements par mois en moyenne n'étaient pas dispensés pour des raisons de coûts.

\section{Bonne qualité avec quelques distorsions}

Une nette majorité de médecins hospitaliers estime que la prise en charge des patients est bonne à très bonne dans leur domaine de travail immédiat. Par rapport à 2012 en soins somatiques aigus, moins de médecins ont observé un transfert de patients polymorbides ou atteints de maladies chroniques vers d'autres institutions. La marge de manœuvre pour le traitement 
Question 1 "Existe-t-il, dans votre service/clinique, des systèmes de rémunération comprenant des composantes salariales variables liées à la performance?"

Question 2 "Votre système d'indemnisation contient-il une composante salariale variable liée à la performance?" Question 3 (s'il y a une composante salariale variable liée à la performance) "S'agit-il pour la composante salariale variable liée à la performance:"

\section{En \%, médecins en soins somatiques aigus/psychiatrie/réadaptation}

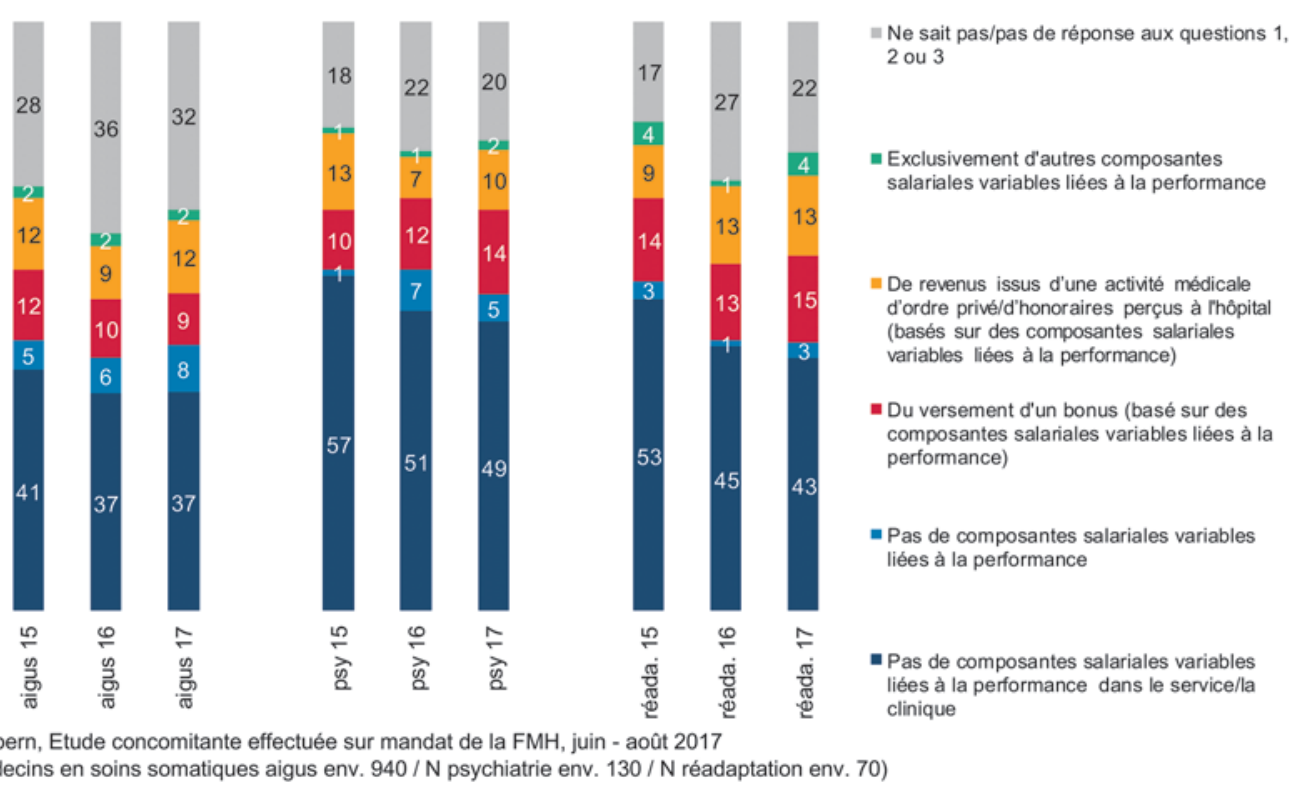

Figure 3: Composantes salariales liées à la performance.

continue majoritairement d'être considérée comme plutôt importante ou très importante. Comme pour les années précédentes, $15 \%$ des médecins hospitaliers en soins somatiques aigus pensent cependant que les caisses-maladie exercent une influence forte à très forte sur le traitement. Ils sont $40 \%$ en réadaptation et $26 \%$ en psychiatrie à partager le même point de vue. En 2017, les médecins en réadaptation hospitalière ont relevé dans leur domaine de travail que les caisses-maladie avaient ralenti en moyenne au moins 7 fois par mois un transfert vers une clinique de réadaptation. $24 \%$ des médecins en cabinet estiment que la sortie après une hospitalisation en soins somatiques aigus se fait souvent trop tôt.

Correspondance: FMH

Baslerstrasse 47 CH-4600 Olten Tél. 0313591111 Fax 0313591112 tarife.spital[at]fmh.ch

\section{Continuer d'observer - agir contre les effets indésirables}

Indépendamment de savoir quelles modifications du système de santé actuellement discutées seront effec- tivement mises en œuvre, il est important d'analyser suffisamment tôt et sur le long terme les effets possibles. Car des effets secondaires tels que l'augmentation des charges administratives entraînent des coûts importants. L'optimisation des processus et une bonne collaboration avec les caisses-maladie sont de ce fait indispensables. Non seulement cela revêt la plus haute importance pour la satisfaction des médecins mais également pour le bien-être des patients.

\section{Séminaires de formation continue}

La FMH et $\mathrm{H}+-$ Bildung continuent de proposer leurs séminaires en allemand pour répondre à la forte demande:

Série de séminaires sur la conduite et le management pour médecins

Médecine et économie: séminaire intensif pour médecins

Pour en savoir plus: www.fmh.ch $\rightarrow$ Tarifs hospitaliers. 